PROCEEDINGS OF THE

AMERICAN MATHEMATICAL SOCIETY

Volume 132, Number 6, Pages 1659-1666

S 0002-9939(04)07436-2

Article electronically published on January 29, 2004

\title{
WEAK COMPACTNESS IS EQUIVALENT TO THE FIXED POINT PROPERTY IN $c_{0}$
}

\author{
P. N. DOWLING, C. J. LENNARD, AND B. TURETT
}

(Communicated by Jonathan M. Borwein)

\begin{abstract}
A nonempty, closed, bounded, convex subset of $c_{0}$ has the fixed point property if and only if it is weakly compact.
\end{abstract}

\section{INTRODUCTION}

In 1981, B. Maurey 9], E. Odell and Y. Sternfeld [11], and R. Haydon, E. Odell and Y. Sternfeld [6] published results on the existence of fixed points of nonexpansive maps on subsets of $c_{0}$. The most general result of these was due to Maurey who used ultrapower techniques to prove that nonempty, weakly compact, convex subsets of $c_{0}$ have the fixed point property. That is, every nonexpansive mapping of a nonempty, weakly compact, convex subset of $c_{0}$ into itself has a fixed point.

Recently there have been several articles investigating the converse of Maurey's theorem. In 1998, E. Llorens-Fuster and B. Sims [8] showed that the closed, bounded, convex subsets of $c_{0}$ with nonempty interior fail to have the fixed point property, and that there exist nonempty convex subsets of $c_{0}$ that are compact in a topology slightly weaker than the weak topology that also fail to have the fixed point property. (Recently, M. Japón Pineda [7] extended this second result to Banach spaces containing $c_{0}$.) Llorens-Fuster and Sims' investigations led them to conjecture that closed, bounded, convex subsets of $c_{0}$ with the fixed point property are weakly compact.

Partial results along these lines have been obtained in Domínguez Benavides, Japón Pineda and Prus [3] and Dowling, Lennard and Turett [4. In both of these articles, the authors provide characterizations of the weakly compact convex subsets of $c_{0}$ in terms of the fixed point property for certain classes of mappings. For example, in 4, it is shown that a closed, bounded, convex subset of $c_{0}$ is weakly compact if and only if all of its nonempty, closed, convex subsets have the fixed point property for nonexpansive mappings. However, this result is not strong enough to prove the conjecture of Llorens-Fuster and Sims. The result only guarantees that a closed, bounded, convex subset $K$ of $c_{0}$ that is not weakly compact contains a further closed, bounded, convex subset $K_{0}$ that fails the fixed point property. It

Received by the editors June 11, 2002.

2000 Mathematics Subject Classification. Primary 47H10, 47H09, 46E30.

The second author thanks Paddy Dowling and the Department of Mathematics and Statistics at Miami University for their hospitality during part of the preparation of this paper. He also acknowledges the financial support of Miami University. 
does not guarantee that the set $K$ itself fails the fixed point property. Thus, the following theorem both improves and clarifies the situation.

Theorem 1. Let $K$ be a nonempty, closed, bounded, convex subset of $\left(c_{0},\|\cdot\|_{\infty}\right)$. Then $K$ is weakly compact if and only if $K$ has the fixed point property ; i.e., every nonexpansive mapping $U: K \longrightarrow K$ has a fixed point.

Moreover, if $K$ is non-weakly compact, there exists a contractive mapping $T$ : $K \longrightarrow K$ (i.e., $\|T(u)-T(v)\|_{\infty}<\|u-v\|_{\infty}$ for all $u, v \in K$ with $u \neq v$ ), such that $T$ is fixed point free.

Thus, as conjectured by Llorens-Fuster and Sims, the converse of Maurey's $c_{0}$ theorem is true. We remark that our result holds when the underlying scalar field is $\mathbb{R}$ and also when it is $\mathbb{C}$.

The proof of the theorem depends on the notions of asymptotically isometric $c_{0}$ basic sequences and asymptotically isometric $c_{0}$-summing basic sequences. Recall from [4] that a sequence $\left(y_{n}\right)_{n \in \mathbb{N}}$ in a Banach space $X$ is an asymptotically isometric $c_{0}$-summing basic sequence if there exists a null sequence $\left(\varepsilon_{n}\right)_{n \in \mathbb{N}}$ in $(0, \infty)$ such that

$$
\sup _{n \in \mathbb{N}}\left(\frac{1}{1+\varepsilon_{n}}\right)\left|\sum_{j=n}^{\infty} t_{j}\right| \leq\left\|\sum_{n=1}^{\infty} t_{n} y_{n}\right\| \leq \sup _{n \in \mathbb{N}}\left(1+\varepsilon_{n}\right)\left|\sum_{j=n}^{\infty} t_{j}\right|,
$$

for all $\left(t_{n}\right)_{n \in \mathbb{N}} \in c_{00}$, the space of finitely nonzero sequences. Recall also that if a sequence $\left(y_{n}\right)_{n \in \mathbb{N}}$ is an asymptotically isometric $c_{0}$-summing basic sequence and if the sequence $\left(w_{n}\right)_{n \in \mathbb{N}}$ is defined by $w_{n}:=y_{n}-y_{n-1}$, where $y_{0}:=0$, then for all $\left(t_{n}\right)_{n \in \mathbb{N}} \in c_{0}$

$$
\sup _{n \in \mathbb{N}}\left(\frac{1}{1+\varepsilon_{n}}\right)\left|t_{n}\right| \leq\left\|\sum_{n=1}^{\infty} t_{n} w_{n}\right\| \leq \sup _{n \in \mathbb{N}}\left(1+\varepsilon_{n}\right)\left|t_{n}\right| .
$$

As in 4, a sequence $\left(w_{n}\right)_{n \in \mathbb{N}}$ satisfying $(*)$ is called an asymptotically isometric $c_{0}$-basic sequence. For information concerning asymptotically isometric $c_{0}$-basic sequences and asymptotically isometric $c_{0}$-summing basic sequences, see 4 ] and the references therein. The following result (Theorem 4 in [4]) as well as certain technical details in its proof will prove crucial.

Theorem 2. 4] Let $K$ be a closed, bounded, convex subset of $\left(c_{0},\|\cdot\|_{\infty}\right)$ that is not weakly compact. Then $K$ contains a nonzero multiple of an asymptotically isometric $c_{0}$-summing basic sequence.

Thus, if $K$ is a closed, bounded, convex subset of $c_{0}$ that is not weakly compact, then there exists $L>0$ and an asymptotically isometric $c_{0}$-summing basic sequence $\left(y_{n}\right)_{n \in \mathbb{N}}$ in $c_{0}$ such that the sequence $\left(L y_{n}\right)_{n \in \mathbb{N}}$ is in $K$. With $K_{0}:=\overline{\operatorname{co}}\left\{y_{n}\right\}$, it is easy to see that there exists a nonexpansive map $T: L K_{0} \rightarrow L K_{0}$ without a fixed point if and only if there exists a nonexpansive map $\widetilde{T}: K_{0} \rightarrow K_{0}$ without a fixed point. Merely define $\widetilde{T}$ as the composition of the maps: multiplication by $L, T$, and multiplication by $1 / L$. Thus, in utilizing the theorem to prove Theorem 1 , it suffices to assume that $L=1$; i.e., that $K$ contains an asymptotically isometric $c_{0}$-summing basic sequence.

The technical detail from the proof of the above theorem that will be used in the proof of Theorem 1 is a specific property of the asymptotically isometric $c_{0^{-}}$ basic sequence $\left(w_{n}\right)_{n \in \mathbb{N}}$ constructed in [4]. In particular, there exists a strictly 
increasing sequence $\left(N_{M(n)}\right)_{n \geq 0}$ in $\mathbb{N} \cup\{0\}$ such that $N_{M(0)}:=0$ and the elements $w_{n}=\left(w_{i}^{n}\right)=\left(y_{i}^{n}-y_{i}^{n-1}\right)$ satisfy

$$
\max _{N_{M(n-1)}<i \leq N_{M(n)}}\left|\sum_{k=1}^{\infty} t_{k} w_{i}^{k}\right| \geq\left|t_{n}\right|-\left(\frac{\delta}{4^{n-2}}\right)\|t\|_{\infty}
$$

for all $n \in \mathbb{N}$ and all $\left(t_{k}\right)_{k \in \mathbb{N}} \in c_{0}$. Here $\delta \in\left(0,4^{-7}\right)$ is a constant.

We remark that this condition ( $)$ is crucial in [4] for establishing the left inequality in $(*)$ above, so that $(\mathbf{N})$ and the right inequality in $(*)$ provide us with a sharpening of Theorem 2 .

\section{Proof of Theorem 1}

Proof. Since one direction is just a restatement of Maurey's result, it is only necessary to show that, if $K$ is not weakly compact, then there exists a nonexpansive self-map $T$ of $K$ without a fixed point. Indeed, we will show that there exists a contractive such $T$, i.e., $\|T(u)-T(v)\|_{\infty}<\|u-v\|_{\infty}$ for all $u, v \in K$ with $u \neq v$.

Fix a closed, bounded, convex subset $K$ of $c_{0}$ that is not weakly compact. By our comments above, we may assume that $K$ contains an asymptotically isometric $c_{0}$-summing basic sequence $\left(y_{n}\right)_{n \in \mathbb{N}}$ where there is no loss in generality in assuming that $\varepsilon_{n}<2^{-1} \cdot 4^{-n}$, for all $n \geq 2$. Then $\left(w_{n}\right)_{n \in \mathbb{N}}$ defined by $w_{n}:=y_{n}-y_{n-1}$, where $y_{0}:=0$, is an asymptotically isometric $c_{0}$-basic sequence. Defining the closed, convex subset $K_{0}:=\overline{\mathrm{co}}\left\{y_{n}\right\}$ of $K$ and using $y_{n}=w_{1}+\cdots+w_{n}$ for $n \in \mathbb{N}$ yields:

$$
K_{0}=\overline{\mathrm{co}}\left\{y_{n}\right\}=\left\{\sum_{n=1}^{\infty} t_{n} w_{n}:\left(t_{n}\right)_{n \in \mathbb{N}} \in c_{0}, 1=t_{1} \geq t_{2} \geq \cdots \geq 0\right\} .
$$

We begin by defining a nonexpansive map $S$ from $c_{0}$ into $K_{0}$ as a composition of four mappings: firstly $R: c_{0} \longrightarrow c_{0}^{\downarrow}$, followed by $J: c_{0}^{\downarrow} \longrightarrow A$, then $V: A \longrightarrow K_{0}$, and finally $M: K_{0} \longrightarrow K_{0}$. Here,

$$
\begin{gathered}
c_{0}^{\downarrow}:=\left\{s=\left(s_{n}\right)_{n \in \mathbb{N}} \in c_{0}: s_{1} \geq s_{2} \geq s_{3} \geq \cdots \geq 0\right\} \text { and } \\
A:=\left\{t=\left(t_{n}\right)_{n \in \mathbb{N}} \in c_{0}: 1=t_{1} \geq t_{2} \geq \cdots \geq 0\right\}=\left\{t \in c_{0}^{\downarrow}: t_{1}=1\right\} .
\end{gathered}
$$

(Note that the sets $A$ and $K_{0}$ coincide when each $w_{n}=e_{n}$; i.e., $\left(y_{n}\right)_{n \in \mathbb{N}}$ is the usual summing basis of $c_{0}$.) We will successively define and discuss each mapping below.

For all $u=\left(u_{1}, u_{2}, \ldots\right) \in c_{0}$, let $R(u):=u^{*}=\left(u_{1}^{*}, u_{2}^{*}, \ldots\right) \in c_{0}$ be the decreasing (i.e., non-increasing) rearrangement of $u$; i.e., $u^{*}=\left(\left|u_{\rho(1)}\right|,\left|u_{\rho(2)}\right|, \ldots\right)$ for some one-to-one mapping $\rho: \mathbb{N} \rightarrow \mathbb{N}$ such that $u_{1}^{*} \geq u_{2}^{*} \geq u_{3}^{*} \geq \cdots$. Note that $u^{*} \in c_{0}^{\downarrow}$.

Although the basic properties of $R$ are well known, we shall derive those that we use herein, for the sake of completeness. Fix $u=\left(u_{n}\right)_{n \in \mathbb{N}} \in c_{0}$. Define $u_{1}^{*}:=$ $\max _{n \in \mathbb{N}}\left|u_{n}\right|$, which equals $\left|u_{n_{1}}\right|$ for some $n_{1} \in \mathbb{N}$. We may assume that $n_{1}$ is minimal with this property. Next, $u_{2}^{*}:=\max \left\{\left|u_{n}\right|: n \in \mathbb{N} \backslash\left\{n_{1}\right\}\right\}=\left|u_{n_{2}}\right|$ for some minimal $n_{2} \in \mathbb{N} \backslash\left\{n_{1}\right\}$. Generally, for each $k \in \mathbb{N}$, we inductively define

$$
u_{k+1}^{*}:=\max \left\{\left|u_{n}\right|: n \in \mathbb{N} \backslash\left\{n_{1}, \ldots, n_{k}\right\}\right\},
$$

which equals $\left|u_{n_{k+1}}\right|$ for some minimal $n_{k+1} \in \mathbb{N} \backslash\left\{n_{1}, \ldots, n_{k}\right\}$. It is easy to see from this definition that $u^{*}=\left(u_{n}^{*}\right)_{n \in \mathbb{N}}$ is decreasing and belongs to $c_{0}$. (Moreover, the map $\rho: \mathbb{N} \rightarrow \mathbb{N}$ mentioned above is given by $\rho(k):=n_{k}$, for all $k \in \mathbb{N}$.) By the 
definition, it is straightforward to check the following fact: for all $u \in c_{0}$, for each $k \in \mathbb{N}$,

\section{(\%)}

$$
u_{k}^{*}=\min _{F \subseteq \mathbb{N}: \#(F)=k-1} \max _{n \in \mathbb{N} \backslash F}\left|u_{n}\right| .
$$

As a direct consequence of this min-max characterization, it follows that for all $u, v \in c_{0}$,

$$
\left|u_{k}^{*}-v_{k}^{*}\right| \leq\|u-v\|_{\infty}, \forall k \in \mathbb{N}
$$

Therefore, for all $u, v \in c_{0},\left\|u^{*}-v^{*}\right\|_{\infty} \leq\|u-v\|_{\infty}$. Hence, rearrangement is a nonexpansive mapping on $c_{0}$.

Let us now consider the second mapping $J$. Using an idea of Llorens-Fuster and Sims [8, The proof of Proposition 1], we define $J: c_{0}^{\downarrow} \longrightarrow A$ by

$$
J(s):=\left(1, s_{1} \wedge 1, s_{2} \wedge 1, s_{3} \wedge 1, \ldots\right), \text { for all } s=\left(s_{n}\right)_{n \in \mathbb{N}} \in c_{0}^{\downarrow} .
$$

The following fact is well known (see, for example, [10, Theorem 1.1.1 (x)]): for all $r, s, t \in \mathbb{R}$,

$$
|s-t|=|s \vee r-t \vee r|+|s \wedge r-t \wedge r|
$$

Consequently, it is easy to see that $J$ is nonexpansive on $c_{0}^{\downarrow}$.

The third mapping $V: A \longrightarrow K_{0}$ is formed by taking each $t=\left(t_{n}\right)_{n \in \mathbb{N}} \in A$ to $\sum_{k=1}^{\infty} t_{k} w_{k} \in K_{0}$. Lastly, $M: K_{0} \longrightarrow K_{0}$ is the identity averaged together with the iterates of a right shift operator on $K_{0}$. Indeed, define $Q: K_{0} \longrightarrow K_{0}$ by

$$
Q(\sigma):=w_{1}+t_{1} w_{2}+t_{2} w_{3}+\cdots+t_{n} w_{n+1}+\cdots, \text { for all } \sigma=\sum_{k=1}^{\infty} t_{k} w_{k} \in K_{0} .
$$

Also, let $I$ be the identity operator on $K_{0}$ and $Q^{2}:=Q \circ Q, Q^{3}:=Q \circ Q \circ Q$, and so on. Next, we define $M: K_{0} \longrightarrow K_{0}$ by

$$
M:=\frac{1}{2} I+\frac{1}{2^{2}} Q+\frac{1}{2^{3}} Q^{2}+\frac{1}{2^{4}} Q^{3}+\cdots .
$$

Now, putting everything together, define $S: c_{0} \longrightarrow K_{0}$ by $S:=M \circ V \circ J \circ R=$ $M V J R$. Furthermore, let us introduce some useful notation. Put $\widetilde{u}:=u^{*} \wedge 1=$ $\left(u_{n}^{*} \wedge 1\right)_{n \in \mathbb{N}}$, for all $u \in c_{0}$. Note that for each $u \in c_{0}, J R(u)=\left(1, \widetilde{u}_{1}, \widetilde{u}_{2}, \widetilde{u}_{3}, \ldots\right)$. Then for all $u \in c_{0}$,

$$
\begin{aligned}
S(u)= & M V(J R(u))=M\left(V\left(1, \widetilde{u}_{1}, \widetilde{u}_{2}, \widetilde{u}_{3}, \ldots\right)\right) \\
= & M\left(w_{1}+\widetilde{u}_{1} w_{2}+\widetilde{u}_{2} w_{3}+\cdots+\widetilde{u}_{n} w_{n+1}+\cdots\right) \\
= & \frac{1}{2}\left(w_{1}+\widetilde{u}_{1} w_{2}+\widetilde{u}_{2} w_{3}+\widetilde{u}_{3} w_{4}+\widetilde{u}_{4} w_{5}+\cdots\right) \\
& +\frac{1}{2^{2}}\left(w_{1}+w_{2}+\widetilde{u}_{1} w_{3}+\widetilde{u}_{2} w_{4}+\widetilde{u}_{3} w_{5}+\cdots\right) \\
& +\frac{1}{2^{3}}\left(w_{1}+w_{2}+w_{3}+\widetilde{u}_{1} w_{4}+\widetilde{u}_{2} w_{5}+\widetilde{u}_{3} w_{6}+\cdots\right)+\cdots ; \text { and so } \\
S(u)=w_{1}+ & \left(\frac{1}{2}+\frac{1}{2} \widetilde{u}_{1}\right) w_{2}+\left(\frac{1}{4}+\frac{1}{4} \widetilde{u}_{1}+\frac{1}{2} \widetilde{u}_{2}\right) w_{3}+\cdots \\
& +\left(\frac{1}{2^{n-1}}+\frac{1}{2^{n-1}} \widetilde{u}_{1}+\frac{1}{2^{n-2}} \widetilde{u}_{2}+\frac{1}{2^{n-3}} \widetilde{u}_{3}+\cdots+\frac{1}{2} \widetilde{u}_{n-1}\right) w_{n}+\cdots .
\end{aligned}
$$


We will show below that $S$ is contractive on $c_{0}$, using the fact that $\|\widetilde{u}-\widetilde{v}\|_{\infty} \leq$ $\|u-v\|_{\infty}$, for all $u, v \in c_{0}$. Fix $u, v \in c_{0}$ with $u \neq v$. Define $\alpha_{j}:=\widetilde{u}_{j}-\widetilde{v}_{j}$, for all $j \in \mathbb{N}$. From above,

$$
\begin{aligned}
S(u)-S(v) & =\frac{1}{2} \alpha_{1} w_{2}+\left(\frac{1}{4} \alpha_{1}+\frac{1}{2} \alpha_{2}\right) w_{3}+\cdots \\
& +\left(\frac{1}{2^{n-1}} \alpha_{1}+\frac{1}{2^{n-2}} \alpha_{2}+\cdots+\frac{1}{2} \alpha_{n-1}\right) w_{n}+\cdots
\end{aligned}
$$

and using the rightmost inequality in $(*)$ above, together with the fact that $\varepsilon_{n}<$ $2^{-1} 4^{-n}$ for all $n \geq 2$, it follows that

$$
\begin{aligned}
& \|S(u)-S(v)\|_{\infty} \leq \sup _{n \geq 2}\left(1+\varepsilon_{n}\right)\left|\frac{1}{2^{n-1}} \alpha_{1}+\frac{1}{2^{n-2}} \alpha_{2}+\cdots+\frac{1}{2} \alpha_{n-1}\right| \\
& \leq \sup _{n \geq 2}\left(1+\varepsilon_{n}\right)\left(\frac{1+2 \varepsilon_{2}}{2^{n-1}} \frac{1}{1+2 \varepsilon_{2}}\left|\alpha_{1}\right|+\frac{1+2 \varepsilon_{3}}{2^{n-2}} \frac{1}{1+2 \varepsilon_{3}}\left|\alpha_{2}\right|+\ldots\right. \\
& \left.+\frac{1+2 \varepsilon_{n}}{2^{1}} \frac{1}{1+2 \varepsilon_{n}}\left|\alpha_{n-1}\right|\right) \\
& \leq \max _{m \geq 1} \frac{1}{1+2 \varepsilon_{m+1}}\left|\alpha_{m}\right| \cdot B, \text { where } \\
& B:=\sup _{n \geq 2}\left(1+\varepsilon_{n}\right)\left(\frac{1+2 \varepsilon_{2}}{2^{n-1}}+\frac{1+2 \varepsilon_{3}}{2^{n-2}}+\cdots+\frac{1+2 \varepsilon_{n}}{2^{1}}\right) \\
& \leq \sup _{n \geq 2}\left(1+\frac{1}{2\left(4^{n}\right)}\right)\left(\left[\frac{1}{2^{n-1}}+\frac{1}{2^{n-2}}+\cdots+\frac{1}{2^{1}}\right]\right. \\
& \left.+\left[\frac{1}{4^{2} 2^{n-1}}+\frac{1}{4^{3} 2^{n-2}}+\cdots+\frac{1}{4^{n} 2^{1}}\right]\right) \\
& =\sup _{n \geq 2}\left(1+\frac{1}{2\left(4^{n}\right)}\right)\left(1-\frac{1}{2^{n-1}}+\left[\frac{1}{2^{n+3}}+\frac{1}{2^{n+4}}+\cdots+\frac{1}{2^{2 n+1}}\right]\right) \\
& \leq \sup _{n \geq 2}\left(1+\frac{1}{4^{n}}\right)\left(1-\frac{1}{2^{n-1}}+\frac{1}{2^{n+2}}\right) \leq \sup _{n \geq 2}\left(1+\frac{1}{4^{n}}-\frac{7}{2^{n+2}}\right)=1 \text {. }
\end{aligned}
$$

So,

$$
\|S(u)-S(v)\|_{\infty} \leq \max _{m \geq 1} \frac{\left|\widetilde{u}_{m}-\widetilde{v}_{m}\right|}{1+2 \varepsilon_{m+1}} .
$$

Recall that $u \neq v$. If $\widetilde{u}=\widetilde{v}$, then $\|S(u)-S(v)\|_{\infty}=0<\|u-v\|_{\infty}$. Otherwise, $\widetilde{u} \neq \widetilde{v}$, which implies

$$
\|S(u)-S(v)\|_{\infty} \leq \max _{m \geq 1} \frac{\left|\widetilde{u}_{m}-\widetilde{v}_{m}\right|}{1+2 \varepsilon_{m+1}}<\max _{m \geq 1}\left|\widetilde{u}_{m}-\widetilde{v}_{m}\right|=\|\widetilde{u}-\widetilde{v}\|_{\infty} \leq\|u-v\|_{\infty},
$$

and therefore $\|S(u)-S(v)\|_{\infty}<\|u-v\|_{\infty}$, for all $u, v \in c_{0}$ with $u \neq v$. Thus, $S$ is contractive.

Finally, define $T: K \rightarrow K_{0} \subseteq K$ to be the restriction of $S$ to $K$. The mapping $T$ is contractive.

We will next show that $T$ fails to have a fixed point in $K$. Observe first that from the definition above, one can readily show that for all $u \in c_{0}$, for each $k \in \mathbb{N}$,

$$
u_{k}^{*}=\max _{G \subseteq \mathbb{N}: \#(G)=k} \min _{n \in G}\left|u_{n}\right| .
$$


Now assume, to get a contradiction, that $u \in K$ is a fixed point of $T$ and recall that $0<\delta<4^{-7}$. Then

$$
\begin{aligned}
u & =T(u)=w_{1}+\left(\frac{1+\widetilde{u}_{1}}{2}\right) w_{2}+\left(\frac{1}{4}+\frac{1}{4} \widetilde{u}_{1}+\frac{1}{2} \widetilde{u}_{2}\right) w_{3}+\cdots \\
& +\left(\frac{1}{2^{k-1}} \widetilde{u}_{1}+\frac{1}{2^{k-2}} \widetilde{u}_{2}+\frac{1}{2^{k-3}} \widetilde{u}_{3}+\cdots+\frac{1}{2} \widetilde{u}_{k-1}\right) w_{k}+\cdots \\
& =: \sum_{k=1}^{\infty} t_{k} w_{k} .
\end{aligned}
$$

Applying (\$) with $n=1$ yields that there exists $i_{1} \in\left\{1, \ldots, N_{M(1)}\right\}$ such that

$$
\begin{aligned}
\left|u_{i_{1}}\right| & =\left|(T(u))_{i_{1}}\right|=\left|\sum_{k=1}^{\infty} t_{k} w_{i_{1}}^{k}\right| \\
& \geq\left|t_{1}\right|-\frac{\delta}{4^{1-2}}\|t\|_{\infty}=1-4 \delta .
\end{aligned}
$$

So, $u_{1}^{*} \geq\left|u_{i_{1}}\right| \geq 1-4 \delta$; and thus $\widetilde{u}_{1}=u_{1}^{*} \wedge 1 \geq 1-4 \delta$.

Next, applying ( $)$ with $n=2$, we see that there exists $i_{2} \in\left\{N_{M(1)}+1, \ldots\right.$, $\left.N_{M(2)}\right\}$ such that

$$
\begin{aligned}
\left|u_{i_{2}}\right| & =\left|(T(u))_{i_{2}}\right| \geq\left|t_{2}\right|-\frac{\delta}{4^{2-2}}\|t\|_{\infty}=\frac{1}{2}+\frac{1}{2} \widetilde{u}_{1}-\delta \\
& \geq \frac{1}{2}+\frac{1}{2}(1-4 \delta)-\delta=1-2 \delta-\delta \geq 1-4 \delta .
\end{aligned}
$$

Hence, using $(\diamond)$ above and the fact that $i_{2}>i_{1}$, it follows that $u_{2}^{*} \geq \min \left\{\left|u_{i_{1}}\right|,\left|u_{i_{2}}\right|\right\}$ $\geq 1-4 \delta$; and consequently $\widetilde{u}_{2}=u_{2}^{*} \wedge 1 \geq 1-4 \delta$.

Furthermore, applying $(\boldsymbol{\$})$ with $n=3$ gives that there exists $i_{3} \in\left\{N_{M(2)}+1, \ldots\right.$, $\left.N_{M(3)}\right\}$ such that

$$
\begin{aligned}
\left|u_{i_{3}}\right| & =\left|(T(u))_{i_{3}}\right| \geq\left|t_{3}\right|-\frac{\delta}{4^{3-2}}\|t\|_{\infty}=\frac{1}{4}+\frac{1}{4} \widetilde{u}_{1}+\frac{1}{2} \widetilde{u}_{2}-\frac{\delta}{4} \\
& \geq \frac{1}{4}+\frac{1}{4}(1-4 \delta)+\frac{1}{2}(1-4 \delta)-\frac{\delta}{4} \geq 1-2 \delta-\delta-\frac{\delta}{2} \geq 1-4 \delta .
\end{aligned}
$$

Since $i_{3}>i_{2}>i_{1}$, we may use $(\diamond)$ to see that $u_{3}^{*} \geq \min \left\{\left|u_{i_{1}}\right|,\left|u_{i_{2}}\right|,\left|u_{i_{3}}\right|\right\} \geq 1-4 \delta$; and therefore $\widetilde{u}_{3}=u_{3}^{*} \wedge 1 \geq 1-4 \delta$.

Continuing inductively, it follows that there exists a strictly increasing sequence of positive integers $\left(i_{n}\right)_{n \in \mathbb{N}}$ such that for all $n \in \mathbb{N}$,

$$
\left|u_{i_{n}}\right| \geq 1-2 \delta-\delta-\frac{\delta}{2}-\cdots-\frac{\delta}{2^{n-2}} \geq 1-4 \delta .
$$

It follows from $(\diamond)$ that for all $n \in \mathbb{N}, \widetilde{u}_{n} \geq 1-4 \delta>0$, which contradicts the fact that $\widetilde{u} \in c_{0}$. Thus, $T$ is fixed point free on $K$. This completes the proof of Theorem 1

\section{Some REMARKS AND A FURTHER RESUlT}

The conclusions of Theorem 1 do not hold in every Banach space. Goebel and Kuczumow [5] and Soardi [12] have given examples of closed, bounded, convex, non-weakly compact sets in $\ell^{1}$ and $L^{\infty}[0,1]$ that have the fixed point property, and Dale Alspach [1] has given an example of a weakly compact, convex subset of $L^{1}[0,1]$ failing to have the fixed point property. There are spaces, other than $c_{0}$, 
for which the conclusions of Theorem 1 hold. However, it is unclear if any of these spaces are significantly different from $c_{0}$.

Corollary 3. Let $K$ be a nonempty, closed, bounded, convex subset of $\left(c_{0}(\Gamma),\|\cdot\|_{\infty}\right)$ where $\Gamma$ is uncountable. Then $K$ is weakly compact if and only $K$ has the fixed point property.

Proof. Since it is known [2] that weakly compact convex sets in $c_{0}(\Gamma)$ have the fixed point property for nonexpansive maps, assume that $K$ is not weakly compact. Then $K$ contains a sequence $\left(x_{n}\right)_{n \in \mathbb{N}}$ with no weakly convergent subsequence. Let $\Gamma_{1}$ denote the support of the sequence $\left(x_{n}\right)_{n \in \mathbb{N}}$; that is, $\Gamma_{1}=\{\gamma \in$

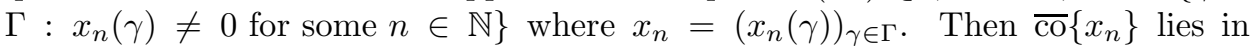
the subspace $c_{0}\left(\Gamma_{1}\right) \times \prod_{\gamma \in \Gamma \backslash \Gamma_{1}}\{0\}$ of $c_{0}(\Gamma)$ and, since $\Gamma_{1}$ is countably infinite, $c_{0}\left(\Gamma_{1}\right) \times \prod_{\gamma \in \Gamma \backslash \Gamma_{1}}\{0\}$ is linearly isometric to $c_{0}$. Thus the previous results can be applied. To be specific, let $P_{1}$ denote the canonical projection from $c_{0}(\Gamma)$ onto $c_{0}\left(\Gamma_{1}\right)$. The closed, bounded, convex, non-weakly compact set $P_{1}(K)$ contains the closed, bounded, convex, non-weakly compact set $\overline{\mathrm{co}}\left\{P_{1}\left(x_{n}\right)\right\}$; and, since this set lies in a closed subspace isometric to $c_{0}, \overline{\mathrm{co}}\left\{P_{1}\left(x_{n}\right)\right\}$ contains (without loss of generality) an asymptotically isometric $c_{0}$-summing basic sequence $\left(y_{n}\right)_{n \in \mathbb{N}}$. Letting $\left(w_{n}\right)_{n \in \mathbb{N}}$ denote the asymptotically isometric $c_{0}$-basic sequence corresponding to $\left(y_{n}\right)_{n \in \mathbb{N}}$ and defining the closed, convex subset $K_{0}:=\overline{\mathrm{co}}\left\{y_{n}\right\}$ implies, as before, that

$$
K_{0}=\overline{\operatorname{co}}\left\{y_{n}\right\}=\left\{\sum_{n=1}^{\infty} t_{n} w_{n}:\left(t_{n}\right)_{n \in \mathbb{N}} \in c_{0}, 1=t_{1} \geq t_{2} \geq \cdots \geq 0\right\} .
$$

If the mapping $S: c_{0} \longrightarrow K_{0}$ is defined as in the proof of Theorem 1 (with $c_{0}\left(\Gamma_{1}\right)$ identified with $c_{0}$ ) and if $T$ is defined as the restriction of $S$ to $P_{1}(K)$, then $T$ is a nonexpansive self-map of $P_{1}(K)$ without a fixed point. Next, with $\overrightarrow{0}$ denoting the zero element in $c_{0}\left(\Gamma \backslash \Gamma_{1}\right)$, note that $y_{n} \times \overrightarrow{0}$ lies in $\overline{\mathrm{co}}\left\{x_{n}\right\}$ and thus is an element of $K$. Therefore, since the range of $T$ is actually a subset of $K_{0}$, the map $U: K \rightarrow K$ defined by $U(x)=\left(T \circ P_{1}(x)\right) \times \overrightarrow{0}$ is a nonexpansive self-map of $K$ without a fixed point and the proof is complete.

\section{REFERENCES}

1. D. Alspach, A fixed point free nonexpansive map, Proc. Amer. Math. Soc. 82(3) (1981), 423-424. MR 82j:47070

2. Jon M. Borwein and Brailey Sims, Nonexpansive mappings on Banach lattices and related topics, Houston J. Math. 10(3) (1984), 339-356. MR 86e:47059

3. T. Domínguez Benavides, M. A. Japón Pineda and S. Prus, Weak compactness and fixed point property for affine mappings, to appear, J. Functional Analysis.

4. P. N. Dowling, C. J. Lennard, and B. Turett, Characterizations of weakly compact sets and new fixed point free maps in $c_{0}$, Studia Math. 154 (2003), no. 3, 277-293. MR 2003m:46016

5. K. Goebel and T. Kuczumow, Irregular convex sets with fixed-point property for non-expansive mappings, Colloq. Math. 40 (1979), 259-264. MR 80j:47068

6. R. Haydon, E. Odell, and Y. Sternfeld, A fixed point theorem for a class of star-shaped sets in $c_{0}$, Israel J. Math. 38(1-2) (1981), 75-81. MR 82c:47070

7. Maria A. Japón Pineda, The fixed-point property in Banach spaces containing a copy of $c_{0}$, Abstract and Applied Analysis 2003 (2003), 183-192, and in "Proceedings of the International Conference on Fixed-Point Theory and Applications", Haifa, June 13-19, 2001 (S. Reich, editor), Hindawi Publishing Corporation, 2003, pp. 183-192.

8. Enrique Llorens-Fuster and Brailey Sims, The fixed point property in $c_{0}$, Canad. Math. Bull. 41(4) (1998), 413-422. MR 99i:47097 
9. B. Maurey, Points fixes des contractions de certains faiblement compacts de $L^{1}$, Seminaire d'Analyse Fonctionelle, 1980-1981, Centre de Mathématiques, École Polytech., Palaiseau, 1981, Exp. No. VIII, 19 pp. MR 83h:47041

10. P. Meyer-Nieberg, Banach Lattices, Springer-Verlag, New York-Berlin-Heidelberg, 1991. MR 93f: 46025

11. E. Odell and Y. Sternfeld, A fixed point theorem in $c_{0}$, Pacific J. Math. 95(1) (1981), 161-177. MR 83b:47060

12. P. M. Soardi, Existence of fixed points on nonexpansive mappings in certain Banach lattices, Proc. Amer. Math. Soc. 73 (1979), 25-29. MR 80c:47051

Department of Mathematics and Statistics, Miami University, Oxford, Ohio 45056

E-mail address: dowlinpn@muohio.edu

Department of Mathematics, University of Pittsburgh, Pittsburgh, Pennsylvania 15260

E-mail address: lennard@pitt.edu

Department of Mathematics and Statistics, Oakland University, Rochester, MichiGAN 48309

E-mail address: turett@oakland.edu 